\title{
EHM Investigation of Hydrogen Adsorption on Model Nickel Substrates
}

\author{
D. L. Lazarov and D. R. Drakova \\ University of Sofia, Faculty of Chemistry, Sofia, Bulgaria
}

Z. Naturforsch. 32 a, 1152-1156 [1977]; received April 20, 1977)

\begin{abstract}
This paper comprises the results of an Extended Hückel investigation of dissociative hydrogen adsorption on optimized models of the three low-index crystal faces of nickel. The frontier MO's approach proves suitable for adsorption studies of this type. Two adsorption forms for hydrogen atoms are established - on top of substrate atoms and in the holes of the substrate. Dissociative non-activated adsorption of hydrogen atoms on top of substrate atoms is found to be energetically most favourable. A correlation between the theoretical and $\beta_{1}$ and $\beta_{2}$ experimental adsorption forms is established. The heat of adsorption diminishes in the order of diminishing density of the crystal face $Q(\mathbf{1 1 1})>Q(\mathbf{1 0 0})>Q(\mathbf{1 1 0})$. There exist experimental data corroborating this result.
\end{abstract}

The interaction of hydrogen with a nickel surface is the subject of many experimental and theoretical studies ${ }^{1-9}$. It is not common, but for several theoretical investigations, that an attempt is made to ascertain the applicability of the methods used and the adequacy of the applied models. Though it is a cumbersome task and the conclusions are often disputable, we consider the adoption of certain criteria for the usefulness of the models necessary in cluster investigations.

The present investigation is an attempt to study the adsorption of atomic and molecular hydrogen on model nickel substrates. Theoretical data are obtained for the adsorption forms on model substrates which are optimized with respect to certain criteria.

The Extended Hückel method is applied to substrate chains of two to eight nickel atoms and twodimensional substrate models of five and seven atoms. It is difficult to point out the most suitable criteria for the cluster approach. We adopt as such certain quantities for which experimental data are available:

1. Specific energy of delocalization [SED]. It is defined as the bonding energy per substrate atom and may be compared with the cohesion energy of nickel $4.4 \mathrm{eV}^{10}$. SED is an increasing function of the number of substrate model atoms N. SED should acquire a constant value for atomic aggregates which are suitable approximations of the massive metal with respect to this criterion.

Reprint requests to Dr. D. L. Lazarov, Faculty of Chemistry, University of Sofia, 1 Anton Ivanov avenue, $B G$-Sofia 26.
2. Orbital energy of the highest occupied molecular orbital $E$ Hомо. This energy level corresponds to the Fermi level at $0 \mathrm{~K}$. $E^{\text {Homo }}$ is expected to have a constant value with respect to $\mathrm{N}$ for the appropriate substrate model.

3. Relative positions of sp- and d-bands. It is known that the wide sp-band overlaps the narrow d-band in nickel.

4. Mean electron population of s- and d-AO's per one substrate atom. The d-band of nickel is known to contain 0.6 vacancies ${ }^{11}$ and 9.4 electrons.

5. d-band width. It is usually approximated with the energy difference between the lowest and highest MO's of predominant d-character. The experimental values vary from $2.5^{13}$ to $3.3 \mathrm{eV} 12$.

The characteristics of the substrate models used should agree with these criteria. Agreement and consistency are necessary with respect to the following variables:

a) Number of substrate atoms in the model $-\mathrm{N}$.

b) Crystallographic direction or plane.

We used the frontier molecular orbitals [FMO] approximation, taking into account the interactions between the valence MO's of the adsorbate $[\mathrm{A}]$ and the substrate [S]. These are the highest occupied and the lowest unoccupied MO's of A and S. This approach allows a considerable reduction of computer time. A theoretical basis for the FMO approximation is presented in the works of Fukui et al. ${ }^{14}$. We tested the possibilities for its application to adsorption processes by carrying out two parallel calculations on the same adsorption system: a) by accounting for the interactions between all mole- 
cular orbitals of $\mathrm{A}$ and $\mathrm{S}, \mathrm{b})$ by using the FMO approximation. The items of chief interest during adsorption, namely the equilibrium adsorption sites and distances, the relative positions of the curves for atomic and molecular adsorption, and the direction of charge transfer are not influenced by the choice of the MO basis set. As it should be expected there are differences in the values of the heats of adsorption, the FMO basis leading to lower values and therefore better agreement with experimental values. We may conclude that using FMO's in the basis is a reasonable approximation in adsorption studies.

The FMO's are represented as linear combinations of atomic orbitals. The coefficients in the linear expansions are calculated using the Extended Hückel method [EHM] in the approximation of Wolfsberg and Helmholz ${ }^{15}$. The main parameters in these calculations are the orbital exponents and the diagonal elements of the effective one-electron Hamiltonian $-\mathrm{H}_{i i}$. They are either varied to fit some experimental data or chosen on the grounds of certain physical considerations. After variation of both parameters we chose Slater's exponents for $\mathrm{Ni}\left(3 \mathrm{~d}^{8} 4 \mathrm{~s}^{2}\right)$ and $\mathrm{H}\left(1 \mathrm{~s}^{1}\right)$. The diagonal matrix elements of the interaction matrix $\mathrm{H}_{i i}$ were put equal to the valence state ionization potentials 16 . They did not differ considerably from the respective values derived from experimental data by Moore ${ }^{17}$.

A variation of the atomic basis set, used to determine the MO's of S, in the cases of a four atoms substrate chain and a five atoms (100) plane was tried. Similar results were obtained for a basis of [3 d, 4s, $4 \mathrm{p}]$ and [ $3 \mathrm{~d}, 4 \mathrm{~s}]$ Slater type atomic orbitals of Ni. The places for equilibrium adsorption, heats of adsorption, charges and bond orders are practically insensitive towards this change in the atomic basis set. These results justify the adoption of the frontier molecular orbitals approach to adsorption processes, the FMO's of the substrate being linear combinations of $3 \mathrm{~d}$ and $4 \mathrm{~s}$ AO's of the nickel atoms.

The solution of the secular equation leads to the eigenvalues and eigenvectors. The Hückel method involves the approximation of the total energy by the orbital energy. The energy of stabilization in a given reaction, which correlates with the heat of the reaction, is then equal to the difference between the orbital energies of the products and the reagents. This approximation results in considerably high absolute values of all energetic quantities - bond energies, heats of adsorption etc. It is a known merit of EHM, however, to reproduce in a satisfactory way the trends in the changes of energy quantities on converting from one system to another. This is a sufficient basis to compare the heats of adsorption on different crystal faces and the stabilities of the different adsorption forms as well.

The data for one-dimensional substrates can be seen in Table 1. The SED values are practically constant for substrates with $N \geqq 4$. No better values are obtained for larger clusters. The lowest occupied molecular orbital has s-character and hence the s-band overlaps the narrow d-band for $N \geqq 6$. $\mathrm{E}^{\text {номо }}$, corresponding to the Fermi energy at $0 \mathrm{~K}$, does not depend notably on the number of substrate atoms. The d-band width has a maximum for $N=4$ or 6 , the calculated values being smaller than the experimental ones. With respect to mean electron population the models built of more than 4 atoms prove suitable.

The adoption of the above criteria indicates that the four to six atoms chain is adequate. An enlargement of the substrates will not lead to better agreement with the characteristics of bulk nickel.

The results for two-dimensional models, as well as experimental data and theoretical data of Fassaert et al. ${ }^{6}$ and Blyholder ${ }^{8}$, are given in Table 2. The characteristics of the models do not differ con-

Table 1. EHM results for one-dimensional model substrates of a nickel f.c.c. crystal. $R_{\mathrm{NiNi}}=2.49 \AA$ in the crystal direction $[110]$.

\begin{tabular}{lllllll}
\hline $\begin{array}{l}\text { Crystal } \\
\text { Direction }\end{array}$ & $N$ & $\begin{array}{l}\text { Atomic basis } \\
\text { set }\end{array}$ & $\begin{array}{l}\text { SED } \\
{[\mathrm{eV}]}\end{array}$ & $\begin{array}{l}\text { EHomo } \\
{[\mathrm{eV}]}\end{array}$ & $\begin{array}{l}\text { d-band } \\
\text { width [eV] }\end{array}$ & $P_{\text {dd }}$ \\
\hline$[110]$ & 8 & $3 \mathrm{~d}, 4 \mathrm{~s}$ & 5.51 & -9.63 & 1.16 & \\
{$[110]$} & 6 & $3 \mathrm{~d}, 4 \mathrm{~s}$ & 5.49 & -9.69 & 1.25 & 9.33 \\
{$[110]$} & 4 & $3 \mathrm{~d}, 4 \mathrm{~s}$ & 5.46 & -9.72 & 1.26 & 9.50 \\
{$[110]$} & 2 & $3 \mathrm{~d}, 4 \mathrm{~s}$ & 4.95 & -9.65 & 0.71 & 9.00 \\
{$[110]$} & 4 & $3 \mathrm{~d}, 4 \mathrm{~s}, 4 \mathrm{p}$ & 5.48 & -9.72 & 1.26 & 9.93 \\
{$[100]$} & 4 & $3 \mathrm{~d}, 4 \mathrm{~s}$ & 5.01 & -9.97 & 0.13 & 9 \\
\hline
\end{tabular}


Table 2. EHM results for two-dimensional model substrates of Ni (111), (100), and (110) faces. For comparison the theoretical data of Fassaert et al. ${ }^{6}$ and Blyholder ${ }^{8}$ and experimental data are listed too.

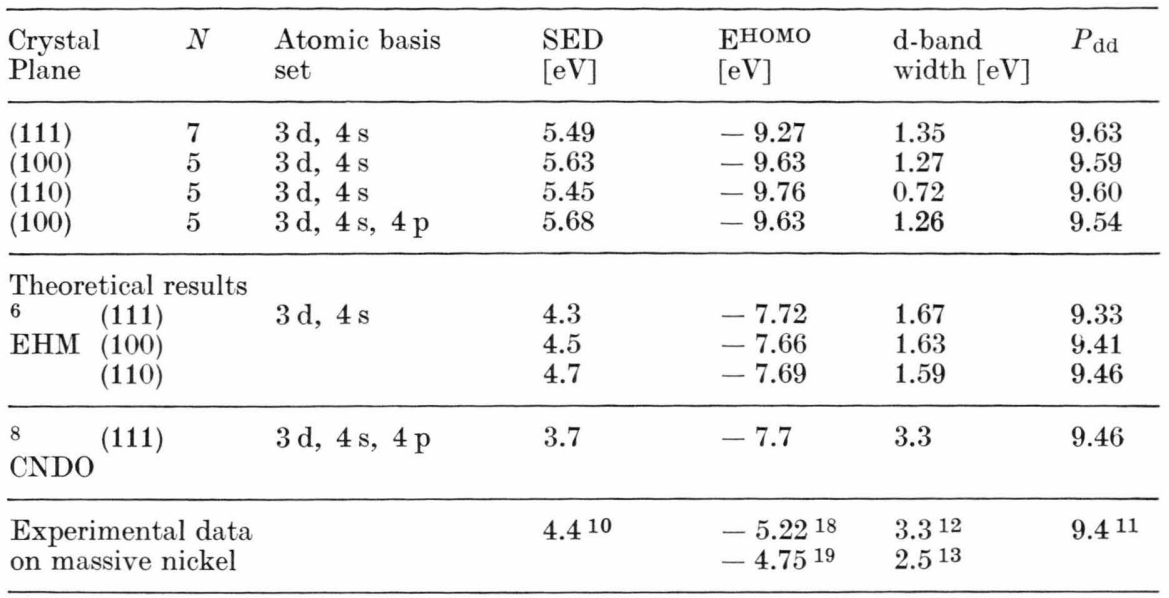

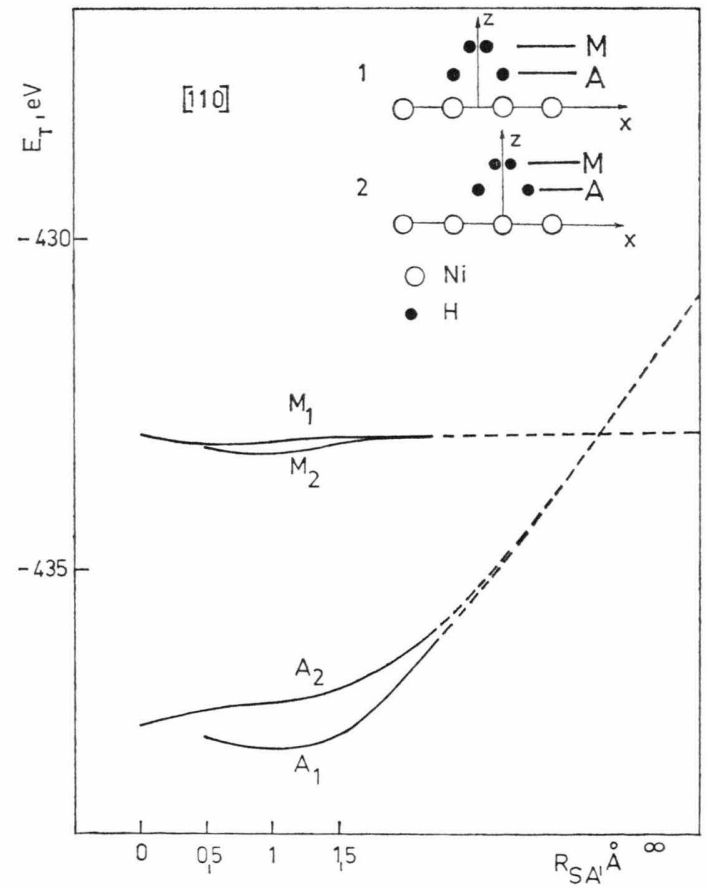

Fig. 1. Total energy of hydrogen molecular [M] and atomic [A] adsorption complexes on one-dimensional model substrates plotted against the adsorbate - substrate distance $R_{\mathrm{SA}}$. Two different mechanisms of adsorbate attack are investigated: (1) - hydrogen atoms situated on top of substrate atoms, (2) - Hydrogen atoms above lattice holes, e.g. $\mathrm{A}_{1}$ indicates atomic adsorption of mechanism (1).

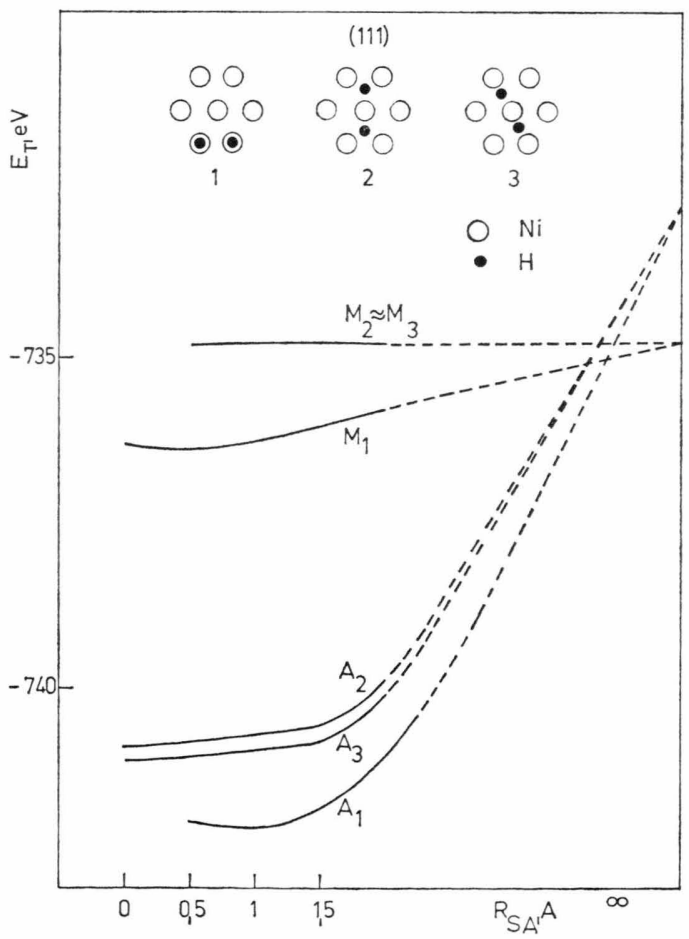

Fig. 2. Total energy of adsorption complexes of hydrogen atoms [A] and a molecule $[\mathrm{M}]$ on $\mathrm{Ni}(\mathbf{1 1 1})$ as a function of $R_{\mathrm{SA}}$. (1), (2), (3) - different mechanisms of adsorbate attack. $\mathrm{A}_{1}$ indicates atomic adsorption according to mechanism (1); $\mathrm{M}_{2}-$ molecular adsorption of mechanism (2), etc. 


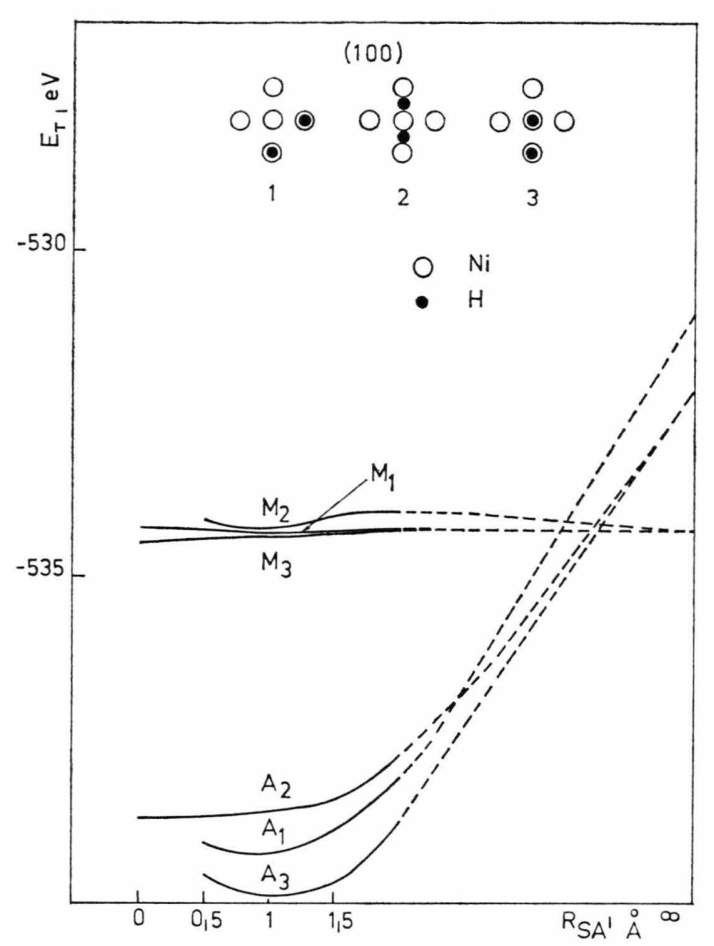

Fig. 3. Total energy of adsorption complexes of hydrogen atoms and a molecule on $\mathrm{Ni}(100)$ plotted against $R_{\mathrm{SA}}$. For explanation of the notation see the text to Figure 2.

siderably from those of the respective chains. This fact justifies their use as models of the different crystal faces for adsorption studies. They yield more possibilities for the orientation of the adsorbate $[\mathrm{A}]$ relative to the substrate $[\mathrm{S}]$.

The model chain for the crystal direction of highest crystallographic density [100] has the best characteristics compared to massive nickel.

Figure 1 gives a plot of the total energy $E_{\mathrm{T}}$ of adsorption complexes as a function of the adsorbate - substrate distance $R_{\mathrm{SA}}$. The figure gives results for adsorption on a four atoms chain and for two different adsorbate attacks -1 and 2,1 referring to attack above atoms of the substrate, $2-$ to attack in the lattice holes. Molecular $\mathrm{M}_{1}$ and $\mathrm{M}_{2}$, and atomic $A_{1}$ and $A_{2}$ adsorption are investigated. The bond distance in $\mathrm{H}_{2}$ is assumed equal to that of $\mathrm{Ni}-\mathrm{Ni}$ in the substrate for adsorption in the form of atoms. The results in Fig. 1 show that dissociative adsorption with hydrogen atoms, situated above the lattice atoms $-\mathrm{A}_{1}-$ is energetically most favourable, $R_{\mathrm{SA}}$ at equilibrium being $1 \AA$. Potential

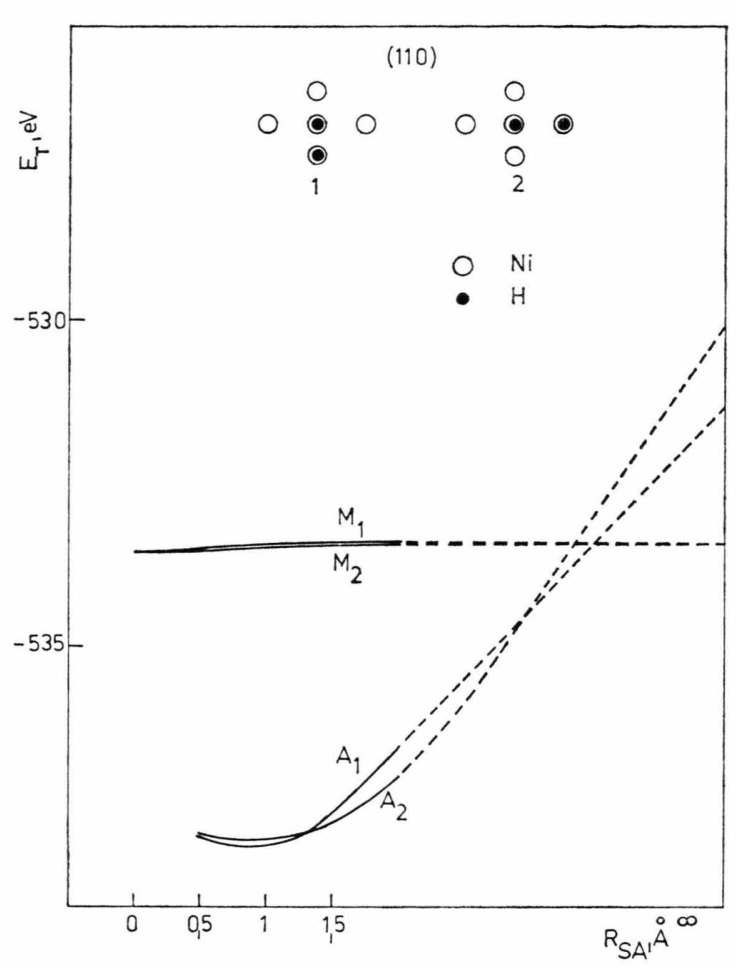

Fig. 4. Total energy of adsorption complexes of atomic and molecular hydrogen on $\mathrm{Ni}(110)$ as a function of $R_{\mathrm{SA}}$. Explanation of the notation may be found in the text to Figure 2.

curves for atomic and molecular adsorption on the two-dimensional models are represented on Figs. 2, 3 and 4 for (111), (100), and (110) respectively. Similar adsorbate attacks are investigated. Nonactivated dissociative chemisorption proves energetically favoured in all cases. Localization of hydrogen atoms above the nickel atoms is most favourable irrespective of the crystal face. $R_{\mathrm{SA}}$ in these cases is also independent on the crystal face and equals $1 \AA$. The heat of adsorption complexes decreases on diminishing the density of the crystal planes $Q(111)>Q(100)>Q(110)$. This result agrees with the data of Christmann ${ }^{21}$ and Lapujoulade 18 who report a similar trend in the isosteric heats of hydrogen adsorption on the three lowindex crystal planes of Ni. Our data indicate that adsorption in the form of molecules is more sensitive towards changes in the substrate. The preferred adsorption sites and $R_{\mathrm{SA}}$ at equilibrium vary for the different crystal faces. The heat of molecular adsorption changes in a similar manner as that of atomic adsorption with the crystal planes. 
The data on the two-dimensional models do not contradict those for the one-dimensional chains. In addition the plane models make possible the investigation of a greater variety of adsorption sites.

The following adsorption forms are obtained in the order of decreasing stability:

1. Dissociative adsorption on top of the substrate atoms.

2. Dissociative adsorption, leading to hydrogen atom incorporation in the substrate surface.

3. Molecular adsorption above nickel atoms.

4. Molecular adsorption above Ni-Ni bonds.

Our data do not allow to choose one of the two molecular adsorption forms as more favourable than the other. There is no experimental evidence to our knowledge, allowing to distinguish between them.

Adsorbed atoms carry negative residual charge, adsorbed molecules are slightly positively polarized.

The theoretically defined adsorption forms can be connected with experimentally determined adsorption forms. Mass-spectrometric and thermodesorption studies ${ }^{1-4,21}$ give evidence that hydrogen is adsorbed on nickel in the form of molecules and atoms, the dissociation during adsorption being non-activated. Adsorbed atoms may exist in two adsorption forms - the more stable $\beta_{2}$ and the less stable $\beta_{1}$ form. $\beta_{2}$ hydrogen atoms can be connected with adsorption on top of $\mathrm{Ni}$ atoms [form 1 above], and $\beta_{1}-$ with hydrogen atoms above holes in the

1 G. Ertl and D. Küppers, Ber. Bunsenges. Phys. Chem. 75, 1017 [1971].

2 G. Wedler and G. Fisch, Ber. Bunsenges. Phys. Chem. 76, 1160 [1972].

3 G. Wedler, G. Fisch, and H. Papp, Ber. Bunsenges. Phys. Chem. 74, 186 [1970].

4 G. Wedler and G. Santelmann, Ber. Bunsenges. Phys. Chem. 75, 1026 [1971].

5 P. W. Selwood, J. Catalysis 42, 148 [1976].

6 J. M. Fassaert, H. Verbeek, and A. van der Avoird, Surface Sci. 29, 501 [1972].

D. J. Fassaert and A. van der Avoird, Surface Sci. 55, 291,313 [1976].

7 D. Shopov, A. Andreev, and D. Petkov, J. Catalysis 13, 123 [1969].

8 G. Blyholder, Surface Sci. 42, 249 [1974].

9 C. F. Melius, J. W. Moskowitz, A. P. Mortola, M. B. Baillie, and M. A. Ratner, Surface Sci. 59, 279 [1976].

10 J. S. Griffith, J. Inorg. Nucl. Chem. 3, 15 [1956].

11 G. C. Bond, Catalysis by Metals. Academic Press, London 1962.

12 D. E. Eastman and J. K. Cashion, Phys. Rev. Letters 27, 1520 [1971]. substrate surface [form 2 above]. The negative charges of $\beta_{1}$ and $\beta_{2} \mathrm{H}$ atoms determined theoretically are in agreement with the data on work function ${ }^{1,20}$ and magnetic susceptibility changes ${ }^{5}$ of $\mathrm{Ni}$, following hydrogen adsorption. The increase in work function reported in 1, 20,21 may be connected with a lowering of the energy of the highest occupied molecular orbital in the adsorption complex with respect to $\mathrm{E}^{\mathrm{HOMO}}$ of the substrate.

The results of the present investigation may be summarized as follows:

1. An optimized model, built of the least possible number of substrate atoms, is chosen on the grounds of several criteria, for which experimental data are available. The basis set of MO's and AO's is considered and its influence on the results for adsorption is analized. The interaction between the frontier MO's of adsorbate and substrate only renders the main effects of the adsorption interaction.

2. Molecular and dissociative non-activated chemisorption of hydrogen atoms occur on the substrate surface. Adsorption on top of $\mathrm{Ni}$ atoms is energetically most favourable. The theoretically determined adsorption forms and charge transfer agree with thermodesorption data, work function, and magnetic susceptibility measurements.

3. Atomic adsorption is most stable on the most closely packed crystal face. The heat of adsorption diminishes on decreasing the density of the crystal plane.

13 S. Hüfner, G. K. Wertheim, and J. H. Wernich, Phys. Rev. B 8, 4511 [1973].

14 K. Fukui, T. Yonezawa, and H. Shingu, J. Chem. Phys. 20, 722 [1952].

K. Fukui, T. Yonezawa, C. Nagata, and H. Shingu, ibid. 22, 1433 [1954].

K. Fukui, H. Kato, and T. Yonezawa, Bill. Chem. Soc. Japan 33, 1197, 1201 [1960]; ibid. 34, 442, 1111 [1961].

15 M. Wolfsberg and L. Helmholz, J. Chem. Phys. 20, 837 [1952].

16 H. Basch, A. Viste, and H. B. Gray, Theor. Chim. Acta 3, 458 [1965].

17 C. Moore. Nat. Bur. Stand. Cire. 1, 467 [1947].

18 J. Lapujoulade and K. S. Neil, J. Chem. Phys. 57, 3535 [1972]; Surface Sci. 35, 288 [1973]; J. Chim. Phys. 70, 798 [1973].

19 R. Gerlach and T. N. Rhodin. The Structure and Chemistry of Solid Surfaces, ed. G. A. Somorjai, Wiley, New York, 1969.

20 T. A, Dechar and F. C. Tompkins. Trans. Faraday Soc. 64, 1915 [1968]; Surface Sci. 8, 165 [1967].

21 K. Christmann, O. Schober, G. Ertl, and M. Neumann, J. Chem. Phys. 60, 4528 [1974]. 